\title{
Sequential Multiple Model Filtering with Interrupted Measurements
}

\author{
By Yong-An ZhANG, ${ }^{1)}$ Di ZHOU ${ }^{2)}$ and Guang-Ren DuAN ${ }^{2)}$ \\ ${ }^{1)}$ School of Astronautics, Beihang University, Beijing, China \\ ${ }^{2)}$ Center for Control and Guidance, Harbin Institute of Technology, Harbin, China
}

(Received January 5th, 2006)

\begin{abstract}
This paper considers the joint detection and filtering problem of discrete-time stochastic systems when the measurements are interrupted in a random fashion. By formulating the measurement interruptions into two-state Markov chains, a sequential multiple model filter is developed from the Bayesian point of view. With a soft switching mechanism, the proposed filter automatically abandons the useless measurements in the interrupted time intervals, and captures the correct measurements for recursive estimation. Compared with the widely used interacting multiple model filter, the new filter has a more simple structure and requires less time for computation. A numerical example shows that the proposed multiple model filter can effectively solve the target tracking problem with interrupted range measurements.
\end{abstract}

Key Words: Kalman Filter, Target Tracking, Joint Detection and Filtering, Multiple Model

\section{Nomenclature}

IMM: interacting multiple model

LOS: line of sight

$N[x ; \hat{x}, P]:$ probability density function of Gaussian random variable $x$ with a mean vector $\hat{x}$ and a variance matrix $P$

RMS: root mean square

$p[x]$ : probability density function of $x$

$p[x \mid y]$ : conditional probability density function of $x$ given $y$

$\operatorname{Pr}[x]$ : probability measure of random variable $x$

$\operatorname{Pr}[x \mid y]$ : condition probability of $x$ given $y$

$\operatorname{sgn}(\cdot)$ : the signum function

$Z^{k}$ : the sequence $\left\{z_{1}, z_{2}, \cdots, z_{k}\right\}$

\section{Introduction}

In the conventional Kalman filtering theory, people generally assume that the plant is modeled as a stochastic state-space model, which consists of a stochastic state equation and a noised measurement equation, with the measurements being accessible during the whole observation interval. However, in some practical applications, the measurements can be interrupted possibly in some time intervals and thus obtained with a probability of less than one. To solve the filtering problem in these cases, some researchers assume that the measurement interruptions can be exactly known to the observer, and concentrate on developing optimal filters for stochastic systems with intermittent measurements. ${ }^{1,2)}$ However, since measurement interruptions are often unknown to the observer, these filters are unsuitable for practical applications. Hence, an additional mechanism should be developed to detect the measurement interruptions for on-line recursive estimation. One loose assumption

(C) 2007 The Japan Society for Aeronautical and Space Sciences is that the switching sequence of the measurement interruptions is expressed in terms of a two-state Markov chain. ${ }^{3-5)}$ This assumption leads to a natural Bayesian framework description for the problem. However, this assumption also has some limitations. First, it is possible that only a few measurement components are intermittently observed, and some other measurement components are still obtainable almost surely. Second, different measurement components may have different intermittent properties. Therefore, unlike the assumption that the interruption sequence of the whole measurement is a two-state Markov chain, we give a more reasonable assumption that different measurement components obey different interruption rules. Correspondently, each rule can be described by a finite-state Markov chain.

The filtering problem for stochastic systems with interrupted measurements has potential usefulness in many practical applications. A typical application is the reconfigurable target tracking problem. In active radar-based target tracking applications, the target motion is generally modeled in Cartesian coordinates, while the measurements are carried out in polar coordinates. The relative range and azimuth angle are the most available measurements in a typical radar tracking system. Under certain tactical conditions, the range measurements are possibly interrupted in some time intervals. For example, when serious electronic countermeasures exist, the electronic jam can possibly cause the range measurement to be denied temporarily. ${ }^{6)}$ Improper utilization of the interrupted range measurements can result in divergence of the tracking filter. On the other hand, if the range measurements are discarded completely, the estimation accuracy is inevitably degraded since some uninterrupted range measurements are not used in the tracking filter. Furthermore, weak observability in an angle-only tracking problem can also introduce the divergence of on- 
line estimation when the range measurements are inaccessible, see relevant references for detailed discussions on this topic. ${ }^{6,7)}$ Thus, it is extremely important to make suitable utilization of the partially interrupted range measurements to improve the tracking performance. As we will see in Section 5, such a case can be described by a filtering problem with partially interrupted measurements. Based on our previous work on target tracking with Gaussian mixture measurement noise, ${ }^{8,9)}$ we propose solving this problem using a sequential multiple model filter in a Bayesian framework. Compared with the interacting multiple model filter widely used in numerous applications, ${ }^{10)}$ the proposed filter has a more simplified structure and can be easily implemented in practical applications.

The remainder of this paper is organized as follows. Section 2 is about the problem formulation. Section 3 presents a derivation of the recursive Bayesian solution of the filtering problem. As a linearized Gaussian approximation of the recursive Bayesian solution, a multiple model filter is given in Section 4. As an application example, the target tracking problem with interrupted range measurements is studied in Section 5. Section 6 concludes the paper.

\section{Problem Formulation}

Consider the following stochastic Markovian switching system described by a hybrid state space model:

$$
\left\{\begin{array}{l}
x_{k}=f\left(x_{k-1}\right)+w_{k} \\
z_{k}=\left[\begin{array}{l}
z_{1, k} \\
z_{2, k}
\end{array}\right]=\left[\begin{array}{l}
m_{1, k} h_{1}\left(x_{k}\right)+v_{1, k} \\
m_{2, k} h_{2}\left(x_{k}\right)+v_{2, k}
\end{array}\right],
\end{array}\right.
$$

where $x_{k} \in \mathfrak{R}^{n}$ is the base state, and $f\left(x_{k-1}\right), h_{1}\left(x_{k}\right)$ and $h_{2}\left(x_{k}\right)$ are continuously differentiable functions. The process noise $w_{k} \in \Re^{m}$ is assumed to be white Gaussian with a zero mean and a covariance matrix $E\left[w_{k} w_{k}^{\mathrm{T}}\right]=Q_{k} . z_{k} \in \mathfrak{R}^{p}$ is the measurement consisting of two mutually independent components $z_{1, k} \in \mathfrak{R}^{p_{1}}$ and $z_{2, k} \in \mathfrak{R}^{p_{2}} . v_{k}=\left[v_{1, k}{ }^{\mathrm{T}}, v_{2, k}{ }^{\mathrm{T}}\right]^{\mathrm{T}}$ is white Gaussian measurement noise with a zero mean and a covariance matrix:

$$
E\left[v_{k} v_{k}^{\mathrm{T}}\right]=R_{k}=\left[\begin{array}{cc}
R_{1, k} & 0 \\
0 & R_{2, k}
\end{array}\right] .
$$

$m_{k}=\left\{m_{1, k}, m_{2, k}\right\}$ is the modal state, where $m_{1, k}, m_{2, k} \in\{0,1\}$ are mutually independent modal state components, and $m_{j, k}=0$ represents the interrupted measurement component consists of only noise without state information; while $m_{j, k}=1$ represents the measurement component when correct, $j=1,2$. The modal state components are expressed in terms of two independent two-state Markov chains with pre-specified transition probabilities:

$$
\operatorname{Pr}\left[m_{j, k} \mid m_{1}, m_{2}, \cdots, m_{k-1}\right]=\operatorname{Pr}\left[m_{j, k} \mid m_{j, k-1}\right],
$$

where $m_{j, k} \in\{0,1\}, j=1,2$, and

$$
\sum_{m_{j, k}=0}^{1} \operatorname{Pr}\left[m_{j, k} \mid m_{j, k-1}\right]=1, \quad m_{j, k-1} \in\{0,1\}, \quad j=1,2 .
$$

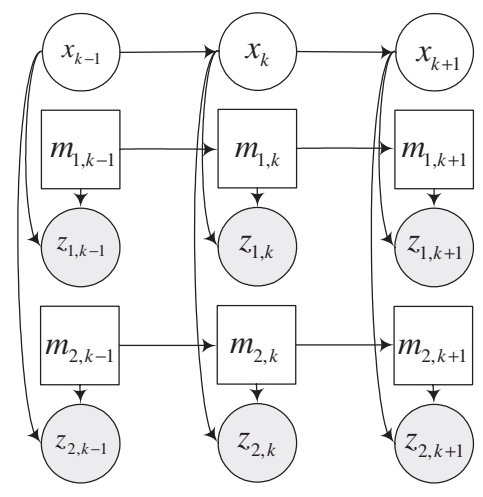

Fig. 1. Dynamic Bayesian network model for the Markovian switching system.

The system can be modeled as the dynamic Bayesian network model depicted in Fig. 1, where square nodes denote discrete modal states, round ones are continuous base states, shaded nodes are measurements, clear nodes are hidden states, and arrows represent allowable transitions with non-zero probability.

We can formulate the cases where a system has more than two classes of measurement interruptions in a similar way. For simplicity, we only discuss the cases of two classes of interruptions. The desired task is to estimate the true base state $x_{k}$ and/or the posterior probability of modal state of the dynamic system from measurements up to time $k$. It is a joint detection and estimation problem where the measurement interruption is detected first, and the base state is then estimated based on the observation sequence and detection of the occurrence of measurement interruptions.

\section{Recursive Bayesian Solution for the Joint Detection and Filtering Problem}

Let $Z^{k}=\left\{z_{1}, z_{2}, \cdots, z_{k}\right\}$ represent the measurements up to time $k$. It can be seen from Eq. (1) that the transition kernel of the base state process $\left\{x_{k}: k=0,1, \cdots\right\}$ is:

$$
p\left[x_{k} \mid x_{k-1}\right]=N\left[x_{k} ; f\left(x_{k-1}\right), Q_{k}\right] .
$$

The likelihoods for the measurement equations of Eq. (1) are given by:

$$
p\left[z_{j, k} \mid x_{k}, m_{j, k}\right]=N\left[z_{k} ; m_{j, k} h_{j}\left(x_{k}\right), R_{j, k}\right],
$$

where $j=1,2$.

Eqs. (5)-(6) are in fact an equivalent probabilistic description of the system represented by Eq. (1). Given these relations, we now aim to find the time recursion of the conditional probability distributions of the base state and modal state.

The recursion process can be divided into two stages. The first stage is the time update stage, which gives the one-stepahead predicted distribution of the state. Notice the Markov properties of the two independent modal state sequences; the one-step-ahead predicted probabilities of the modal state components can be given by: 


$$
\begin{aligned}
\operatorname{Pr} & {\left[m_{j, k} \mid Z^{k-1}\right] } \\
& =\sum_{m_{j, k-1}=0}^{1} \operatorname{Pr}\left[m_{j, k} \mid Z^{k-1}, m_{j, k-1}\right] \operatorname{Pr}\left[m_{j, k-1} \mid Z^{k-1}\right] \\
& =\sum_{m_{j, k-1}=0}^{1} \operatorname{Pr}\left[m_{j, k} \mid m_{j, k-1}\right] \operatorname{Pr}\left[m_{j, k-1} \mid Z^{k-1}\right],
\end{aligned}
$$

where $j=1,2$. Also notice the Markov property of the base state process, we have

$$
\begin{aligned}
p\left[x_{k} \mid Z^{k-1}\right] & \\
& =\int p\left[x_{k}, x_{k-1} \mid Z^{k-1}\right] \mathrm{d} x_{k-1} \\
& =\int p\left[x_{k} \mid x_{k-1}, Z^{k-1}\right] p\left[x_{k-1} \mid Z^{k-1}\right] \mathrm{d} x_{k-1} \\
& =\int p\left[x_{k} \mid x_{k-1}\right] p\left[x_{k-1} \mid Z^{k-1}\right] \mathrm{d} x_{k-1} .
\end{aligned}
$$

Eqs. (7)-(8) imply that, when given the state estimate at time $k-1$, we need not calculate the mixture probabilities in the time update stage. Furthermore, as we know, calculation of the mixture probabilities is a part in the IMM filter. ${ }^{10)}$ Hence, compared with the IMM filter, the time update stage of the recursive solution can be simplified significantly.

Next, we consider the measurement update of the recursive Bayesian solution. Notice the measurement components $z_{1, k}$ and $z_{2, k}$ are mutually independent conditioned on $x_{k}$ and $\left\{m_{1, k}, m_{2, k}\right\}$; i.e.,

$$
p\left[z_{1, k}, z_{2, k} \mid x_{k}, m_{1, k}, m_{2, k}\right]=p\left[z_{1, k} \mid x_{k}, m_{1, k}\right] p\left[z_{2, k} \mid x_{k}, m_{2, k}\right] .
$$

Eq. (9) means that the likelihood function can be decomposed into two parts, each part independently contains information of a measurement component. Hence, the measurement update can be divided into two steps. The first step is the measurement update by $z_{1, k}$. Applying the Bayesian formula, we have

$p\left[x_{k} \mid Z^{k-1}, z_{1, k}, m_{1, k}\right]=\frac{p\left[x_{k} \mid Z^{k-1}, m_{1, k}\right] p\left[z_{1, k} \mid x_{k}, Z^{k-1}, m_{1, k}\right]}{p\left[z_{1, k} \mid Z^{k-1}, m_{1, k}\right]}$.

Since $x_{k}$ and $m_{1, k}$ are mutually independent when only the past measurement information $Z^{k-1}$ is provided, we have:

$$
p\left[x_{k} \mid Z^{k-1}, m_{1, k}\right]=p\left[x_{k} \mid Z^{k-1}\right] .
$$

Hence,

$$
\begin{aligned}
& p\left[x_{k} \mid Z^{k-1}, z_{1, k}, m_{1, k}\right] \\
& =\frac{p\left[x_{k} \mid Z^{k-1}\right] p\left[z_{1, k} \mid x_{k}, Z^{k-1}, m_{1, k}\right]}{p\left[z_{1, k} \mid Z^{k-1}, m_{1, k}\right]} \\
& =\frac{p\left[x_{k} \mid Z^{k-1}\right] p\left[z_{1, k} \mid x_{k}, m_{1, k}\right]}{p\left[z_{1, k} \mid Z^{k-1}, m_{1, k}\right]},
\end{aligned}
$$

where the normalizing constant $p\left[z_{1, k} \mid Z^{k-1}, m_{1, k}\right]$ is given by:

$$
p\left[z_{1, k} \mid Z^{k-1}, m_{1, k}\right]=\int p\left[x_{k} \mid Z^{k-1}\right] p\left[z_{1, k} \mid x_{k}, m_{1, k}\right] \mathrm{d} x_{k} .
$$

Consequently, the conditional probability of mode $m_{1, k}$ given $Z^{k-1}$ and $z_{1, k}$ is:

$$
\operatorname{Pr}\left[m_{1, k} \mid Z^{k-1}, z_{1, k}\right]=\frac{\operatorname{Pr}\left[m_{1, k} \mid Z^{k-1}\right] p\left[z_{1, k} \mid Z^{k-1}, m_{1, k}\right]}{p\left[z_{1, k} \mid Z^{k-1}\right]},
$$

which gives the normalizing constant:

$$
p\left[z_{1, k} \mid Z^{k-1}\right]=\sum_{m_{1, k}=0}^{1} \operatorname{Pr}\left[m_{1, k} \mid Z^{k-1}\right] p\left[z_{1, k} \mid Z^{k-1}, m_{1, k}\right] .
$$

Thus, the condition probability density of the base state after the update of measurement $z_{1, k}$ is:

$$
\begin{aligned}
p & {\left[x_{k} \mid Z^{k-1}, z_{1, k}\right] } \\
& =\sum_{m_{1, k}=0}^{1} \operatorname{Pr}\left[m_{1, k} \mid Z^{k-1}, z_{1, k}\right] p\left[x_{k} \mid Z^{k-1}, z_{1, k}, m_{1, k}\right] .
\end{aligned}
$$

The second step is the measurement update by $z_{2, k}$. Also using the Bayesian formula, we obtain:

$$
\begin{aligned}
p & {\left[x_{k} \mid Z^{k}, m_{2, k}\right] } \\
& =p\left[x_{k} \mid Z^{k-1}, z_{1, k}, z_{2, k}, m_{2, k}\right] \\
& =\frac{p\left[x_{k}, z_{2, k} \mid Z^{k-1}, z_{1, k}, m_{2, k}\right]}{p\left[z_{2, k} \mid Z^{k-1}, z_{1, k}, m_{2, k}\right]} \\
& =\frac{p\left[z_{2, k} \mid x_{k}, Z^{k-1}, z_{1, k}, m_{2, k}\right] p\left[x_{k} \mid Z^{k-1}, z_{1, k}, m_{2, k}\right]}{p\left[z_{2, k} \mid Z^{k-1}, z_{1, k}, m_{2, k}\right]} .
\end{aligned}
$$

Since $x_{k}$ and $m_{2, k}$ are mutually independent when only $Z^{k-1}$ and $z_{1, k}$ are given (see Fig. 1), this implies:

$$
p\left[x_{k} \mid Z^{k-1}, z_{1, k}, m_{2, k}\right]=p\left[x_{k} \mid Z^{k-1}, z_{1, k}\right] .
$$

Notice the fact:

$$
p\left[z_{2, k} \mid x_{k}, Z^{k-1}, z_{1, k}, m_{2, k}\right]=p\left[z_{2, k} \mid x_{k}, m_{2, k}\right],
$$

we obtain:

$$
p\left[x_{k} \mid Z^{k}, m_{2, k}\right]=\frac{p\left[z_{2, k} \mid x_{k}, m_{2, k}\right] p\left[x_{k} \mid Z^{k-1}, z_{1, k}\right]}{p\left[z_{2, k} \mid Z^{k-1}, z_{1, k}, m_{2, k}\right]},
$$

where

$$
\begin{aligned}
& p\left[z_{2, k} \mid Z^{k-1}, z_{1, k}, m_{2, k}\right] \\
& \quad=\int p\left[z_{2, k} \mid x_{k}, m_{2, k}\right] p\left[x_{k} \mid Z^{k-1}, z_{1, k}\right] \mathrm{d} x_{k} .
\end{aligned}
$$

The posterior probability of the mode $m_{2, k}$ conditioned to the measurements $Z^{k}$ is:

$$
\begin{aligned}
\operatorname{Pr} & {\left[m_{2, k} \mid Z^{k}\right] } \\
& =\operatorname{Pr}\left[m_{2, k} \mid Z^{k-1}, z_{1, k}, z_{2, k}\right] \\
& =\frac{\operatorname{Pr}\left[m_{2, k}, z_{2, k} \mid Z^{k-1}, z_{1, k}\right]}{p\left[z_{2, k} \mid Z^{k-1}, z_{1, k}\right]} \\
& =\frac{\operatorname{Pr}\left[m_{2, k} \mid Z^{k-1}, z_{1, k}\right] p\left[z_{2, k} \mid Z^{k-1}, z_{1, k}, m_{2, k}\right]}{p\left[z_{2, k} \mid Z^{k-1}, z_{1, k}\right]} \\
& =\frac{\operatorname{Pr}\left[m_{2, k} \mid Z^{k-1}\right] p\left[z_{2, k} \mid Z^{k-1}, z_{1, k}, m_{2, k}\right]}{p\left[z_{2, k} \mid Z^{k-1}, z_{1, k}\right]},
\end{aligned}
$$

where: 


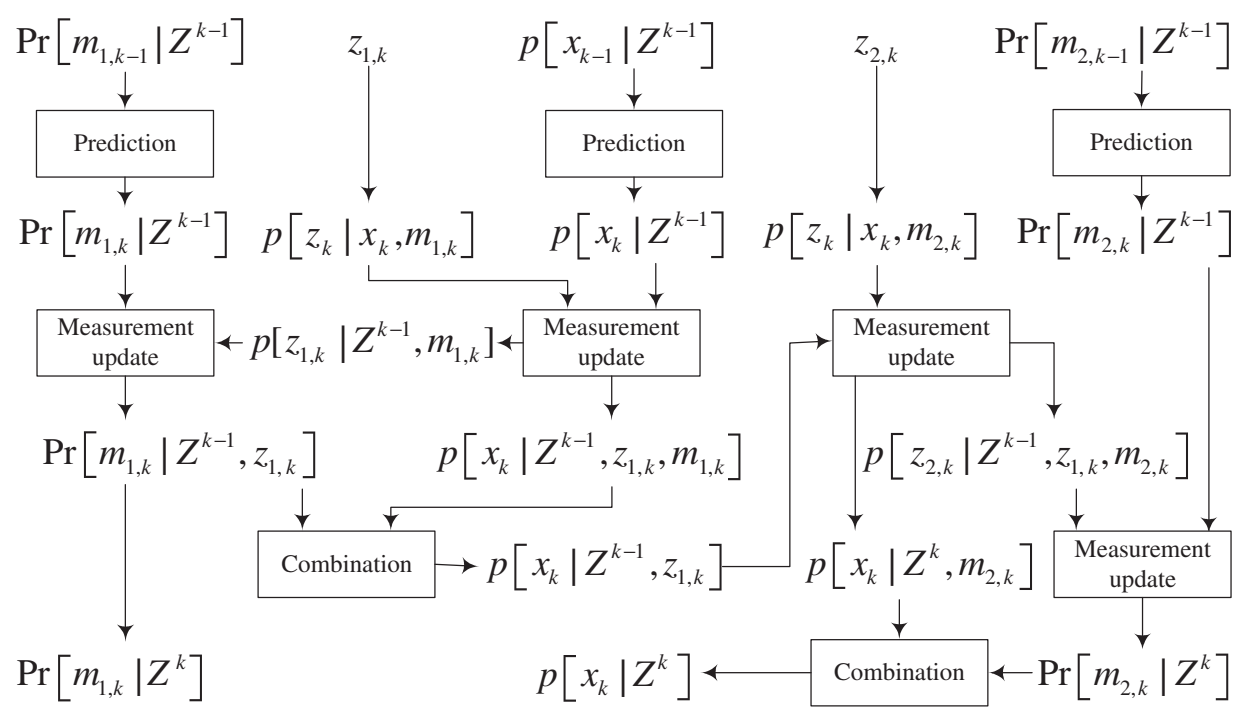

Fig. 2. Recursive relations of the state conditional probability distributions.

$p\left[z_{2, k} \mid Z^{k-1}, z_{1, k}\right]=\sum_{m_{2, k}=0}^{1} \operatorname{Pr}\left[m_{2, k} \mid Z^{k-1}\right] p\left[z_{2, k} \mid Z^{k-1}, z_{1, k}, m_{2, k}\right]$.

Applying the total probability theorem, the posterior probability density function of the base state $x_{k}$ conditioned on $Z^{k}$ can be represented as:

$$
p\left[x_{k} \mid Z^{k}\right]=\sum_{m_{2, k}=0}^{1} \operatorname{Pr}\left[m_{2, k} \mid Z^{k}\right] p\left[x_{k} \mid Z^{k}, m_{2, k}\right] .
$$

Since $m_{1, k}$ and $z_{2, k}$ are mutually independent, it can be seen that Eq. (14) gives the posterior probability distribution of the mode $m_{1, k}$; i.e.,

$$
\begin{aligned}
\operatorname{Pr}\left[m_{1, k} \mid Z^{k}\right] & =\operatorname{Pr}\left[m_{1, k} \mid Z^{k-1}, z_{1, k}, z_{2, k}\right] \\
& =\operatorname{Pr}\left[m_{1, k} \mid Z^{k-1}, z_{1, k}\right] .
\end{aligned}
$$

Thus, we have completed the recursion of the state condition probability distribution. To make it clear, we present a diagram for the recursive relations, as shown in Fig. 2.

\section{Gaussian Approximation of the Recursive Bayesian Solution}

The optimal Bayesian solution in Section 3 does not have an analytical form in practical applications. Even for a linear system, the terms of the base state posterior probability density function in the solution increase exponentially with respect to time. To avoid such an intractable situation, we present a step-by-step linearized Gaussian approximation of the posterior probability density function of the base state.

By linearized Gaussian approximation, we mean that we use a Gaussian distribution to approximate the posterior distribution of the state:

$$
p\left[x_{k} \mid Z^{k}\right] \approx N\left[x_{k} ; \hat{x}_{k \mid k}, P_{k \mid k}\right] .
$$

If we know the base state prior distribution:

$$
p\left[x_{k-1} \mid Z^{k-1}\right]=N\left[x_{k-1} ; \hat{x}_{k-1 \mid k-1}, P_{k-1 \mid k-1}\right],
$$

and the modal state prior probabilities:

$$
\mu_{i, k-1}^{j}=\operatorname{Pr}\left[m_{i, k-1}=j\right], \quad i=1,2, \quad j=0,1,
$$

then by Eqs. (7)-(8), we can obtain a linearized Gaussian predicted base state estimate $\left\{\hat{x}_{k \mid k-1}, P_{k \mid k}\right\}$ by first-order Taylor approximation, as given in Eqs. (29)-(32).

Next, the predicted state estimate is updated using Eqs. (10)-(16), which produces an intermediate base state estimate $\left\{\bar{x}_{k \mid k}, \bar{P}_{k \mid k}\right\}$ and the posterior probabilities of modal state $m_{1, k}$, as shown in Eqs. (33)-(46). Then, the intermediate state estimate $\left\{\bar{x}_{k \mid k}, \bar{P}_{k \mid k}\right\}$ is updated using Eqs. (17)-(24), which yields the final base state estimate $\left\{\hat{x}_{k \mid k}, P_{k \mid k}\right\}$ and the posterior probabilities of the modal state $m_{2, k}$, as shown in Eqs. (47)-(60). Hence, we obtain a sequential multiple model filter, as shown in the following.

\section{Algorithm 1 (Sequential multiple model filter).}

1) Initialization. Pre-specify the a priori estimates of the mean and the covariance of the base state $\left\{\hat{x}_{0 \mid 0}, P_{0 \mid 0}\right\}$, and a priori modal state probabilities $\mu_{i, 0}^{j}=$ $\operatorname{Pr}\left[m_{i, 0}=j\right], i=1,2, j=0,1$.

2) Recursive estimation. $k=1,2, \cdots$

a) Time update.

Time update of the base state estimate:

$$
\begin{gathered}
\hat{x}_{k \mid k-1}=f\left(\hat{x}_{k-1 \mid k-1}\right) \\
A_{k}=\left.\frac{\partial f\left(x_{k-1}\right)}{\partial x_{k-1}}\right|_{x_{k-1}=\hat{x}_{k-1 \mid k-1}} \\
P_{k \mid k-1}=A_{k} P_{k-1 \mid k-1} A_{k}^{\mathrm{T}}+Q_{k} .
\end{gathered}
$$

Time update of the mode probabilities:

$$
\begin{aligned}
& \bar{\mu}_{i, k}^{j}=\sum_{l=0}^{1} \mu_{i, k-1}^{j} \operatorname{Pr}\left[m_{i, k}=j \mid m_{i, k-1}=l\right], \\
& j=0,1, \quad i=1,2 .
\end{aligned}
$$


b) Measurement update by $z_{1, k}$ to produce an intermediate state estimate.

When $m_{1, k}=0$, we have:

$$
\begin{gathered}
\hat{z}_{1, k \mid k-1}^{0}=0, \quad v_{1, k}^{0}=z_{1, k}-\hat{z}_{1, k \mid k-1}^{0}=z_{1, k}, \\
S_{1, k}^{0}=R_{1, k}, \quad K_{1, k}^{0}=0 \\
\bar{x}_{k \mid k}^{0}=\hat{x}_{k \mid k-1}+K_{1, k}^{0} v_{1, k}^{0}=\hat{x}_{k \mid k-1} \\
\bar{P}_{k \mid k}^{0}=P_{k \mid k-1} \\
L_{1, k}^{0}=\frac{1}{\sqrt{\operatorname{det}\left(2 \pi S_{1, k}^{0}\right)}} \exp \left[-\frac{1}{2} v_{1, k}^{0} S_{1, k}^{0}{ }^{-1} v_{r, k}^{0}\right] \\
=\frac{1}{\sqrt{\operatorname{det}\left(2 \pi R_{1, k}\right)}} \exp \left[-\frac{1}{2} z_{1, k}^{\mathrm{T}} R_{1, k}^{-1} z_{1, k}\right] .
\end{gathered}
$$

When $m_{1, k}=1$, we have:

$$
\begin{gathered}
H_{1, k}^{1}=\left.\frac{\partial h_{1}\left(x_{k}\right)}{\partial x_{k}}\right|_{x_{k}=\hat{x}_{k \mid k-1}}, \hat{z}_{1, k \mid k-1}^{1}=h_{1}\left(\hat{x}_{k \mid k-1}\right) \\
v_{1, k}^{1}=z_{1, k}-\hat{z}_{1, k \mid k-1}^{1} \\
S_{1, k}^{1}=H_{1, k}^{1} P_{k \mid k-1} H_{1, k}^{1 \mathrm{~T}}+R_{1, k} \\
K_{1, k}^{1}=P_{k \mid k-1} H_{1, k}^{1 \mathrm{~T}} S_{1, k}^{1}{ }^{1} \\
\bar{x}_{k \mid k}^{1}=\hat{x}_{k \mid k-1}+K_{1, k}^{1} v_{1, k}^{1} \\
\bar{P}_{k \mid k}^{1}=\left(I-K_{1, k}^{1} H_{1, k}^{1}\right) P_{k \mid k-1}\left(I-K_{1, k}^{1} H_{1, k}^{1}\right)^{\mathrm{T}} \\
+K_{1, k}^{1} R_{1, k} K_{1, k}^{1 \mathrm{~T}} \\
L_{1, k}^{1}=\frac{1}{\sqrt{\operatorname{det}\left(2 \pi S_{1, k}^{1}\right)}} \exp \left[-\frac{1}{2} v_{1, k}^{1} \mathrm{~T} S_{1, k}^{1}-1 v_{r, k}^{1}\right]
\end{gathered}
$$

Computation of the posterior probabilities of the modal state $m_{1, k}$ :

$$
\mu_{1, k}^{j}=\operatorname{Pr}\left[m_{1, k} \mid Z^{k}\right]=\frac{L_{1, k}^{j} \bar{\mu}_{1, k}^{j}}{c_{1, k}},
$$

where $j=0,1$, and the normalizing constant $c_{1, k}$ is given by:

$$
c_{1, k}=\sum_{j=0}^{1} L_{1, k}^{j} \bar{\mu}_{1, k}^{j} .
$$

Combination of the mode-based estimates:

$$
\begin{gathered}
\bar{x}_{k \mid k}=\sum_{j=0}^{1} \mu_{1, k}^{j} \bar{x}_{k \mid k}^{j} \\
\bar{P}_{k \mid k}=\sum_{j=0}^{1} \mu_{1, k}^{j}\left[\bar{P}_{k \mid k}^{j}+\left(\bar{x}_{k \mid k}^{j}-\bar{x}_{k \mid k}\right)\left(\bar{x}_{k \mid k}^{j}-\bar{x}_{k \mid k}\right)^{\mathrm{T}}\right] .
\end{gathered}
$$

c) Measurement update by $z_{2, k}$ to produce the modematched base state estimates.

When $m_{2, k}=0$, we have:

$$
\begin{aligned}
& H_{2, k}^{0}=0, v_{2, k}^{0}=z_{2, k}-0=z_{2, k}, \\
& S_{2, k}^{0}=R_{2, k}, K_{2, k}^{0}=0
\end{aligned}
$$

$$
\begin{gathered}
\hat{x}_{k \mid k}^{0}=\bar{x}_{k \mid k}+K_{2, k}^{0} v_{2, k}^{0}=\bar{x}_{k \mid k} \\
P_{k \mid k}^{0}=\bar{P}_{k \mid k} \\
L_{2, k}^{0}=\frac{1}{\sqrt{\operatorname{det}\left(2 \pi S_{2, k}^{0}\right)}} \exp \left[-\frac{1}{2} v_{2, k}^{0}{ }^{\mathrm{T}} S_{2, k}^{0}{ }^{-1} v_{2, k}^{0}\right] \\
=\frac{1}{\sqrt{\operatorname{det}\left(2 \pi R_{2, k}\right)}} \exp \left[-\frac{1}{2} z_{2, k}^{\mathrm{T}} R_{2, k}^{-1} z_{2, k}\right] .
\end{gathered}
$$

When $m_{2, k}=1$, we have:

$$
\begin{gathered}
H_{2, k}^{1}=\left.\frac{\partial h_{2}\left(x_{k}\right)}{\partial x_{k}}\right|_{x_{k}=\bar{x}_{k \mid k}}, \quad \hat{z}_{2, k \mid k-1}^{1}=h_{2}\left(\hat{x}_{k \mid k-1}\right) \\
v_{2, k}^{1}=z_{2, k}-\hat{z}_{2, k}^{1} \\
S_{2, k}^{1}=H_{2, k}^{1} \bar{P}_{k \mid k} H_{2, k}^{1}{ }^{\mathrm{T}}+R_{2, k} \\
K_{2, k}^{1}=\bar{P}_{k \mid k} H_{2, k}^{1}{ }^{\mathrm{T}} S_{2, k}^{1}{ }^{-1} \\
\hat{x}_{k \mid k}^{1}=\bar{x}_{k \mid k}+K_{2, k}^{1} v_{2, k}^{1} \\
P_{k \mid k}^{1}=\left(I-K_{2, k}^{1} H_{2, k}^{1}\right) \bar{P}_{k \mid k}\left(I-K_{2, k}^{1} H_{2, k}^{1}\right)^{\mathrm{T}} \\
+K_{2, k}^{1} R_{2, k} K_{2, k}^{1} \mathrm{~T} \\
L_{2, k}^{1}=\frac{1}{\sqrt{\operatorname{det}\left(2 \pi S_{2, k}^{1}\right)}} \exp \left[-\frac{1}{2} v_{2, k}^{1}{ }^{\mathrm{T}} S_{2, k}^{1}-1 v_{2, k}^{1}\right]
\end{gathered}
$$

Computation of the posterior probabilities of the modal state $m_{2, k}$ :

$$
\mu_{2, k}^{j}=\operatorname{Pr}\left[m_{2, k}=j \mid Z^{k}\right]=\frac{L_{2, k}^{j} \bar{\mu}_{2, k}^{j}}{c_{2, k}},
$$

where $j=0,1$, and the normalizing constant $c_{2, k}$ is given by:

$$
c_{2, k}=\sum_{j=0}^{1} L_{2, k}^{j} \bar{\mu}_{2, k}^{j} .
$$

d) Combination of the mode-based estimates.

$$
\hat{x}_{k \mid k}=\sum_{j=0}^{1} \mu_{2, k}^{j} \hat{x}_{k \mid k}^{j}
$$

$$
P_{k \mid k}=\sum_{j=0}^{1} \mu_{2, k}^{j}\left[\bar{P}_{k \mid k}^{j}+\left(\hat{x}_{k \mid k}^{j}-\hat{x}_{k \mid k}\right)\left(\hat{x}_{k \mid k}^{j}-\hat{x}_{k \mid k}\right)^{\mathrm{T}}\right] \text {. }
$$

We have utilized a two-step measurement update in the above approximated filter. Such a method will bring some advantages in many applications. When the measurement equation consists of both linear (or universally linearizable) and nonlinear components, we can use a two-step algorithm: the first step is a linear or universal linearization measurement update, which introduces an intermediate estimate of the state; and the second step is a nonlinear measurement update based on the intermediate estimate, which produces the final posterior estimate. In this case, the first linear or universal linearization update can provide a more accurate reference point for the second nonlinear measurement up- 


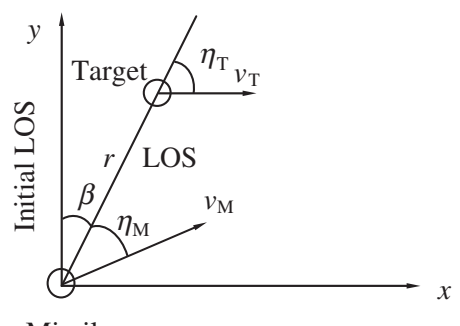

Missile

Fig. 3. Missile-target engagement geometry.

date. ${ }^{11,12)}$ Using this algorithm, we can improve filtering performance. As can be seen, the angle measurement for target tracking applications is, in fact, universally linearizable. $^{12)}$

In order to avoid the underflow phenomenon that often happens in mode probability computation, we implement the computation of mode posterior probability in numerical robust forms. Please see relevant work for details on numerical robust implementation of the multiple model filters. ${ }^{8,13)}$

\section{Target Tracking with Interrupted Range Measure- ments}

Consider a planar radar tracking system with range-bearing measurements in a homing guidance problem. The planar missile-target engagement geometry is depicted in Fig. 3. In the Cartesian coordinates, the state vector $x_{k}$ is composed of the missile-target relative range, relative velocity, and target acceleration; i.e.,

$$
x_{k}=\left[\begin{array}{llllll}
r_{x, k} & r_{y, k} & v_{x, k} & v_{y, k} & a_{\mathrm{T} x, k} & a_{\mathrm{T} y, k}
\end{array}\right]^{\mathrm{T}} .
$$

For the sake of concentrating our attention on the tracking problem, we assume the autopilot of the missile has zero lag. Using the Singer acceleration model, the components of target acceleration are assumed to be independent GaussMarkov stochastic processes. Then the continuous dynamic model is given by:

$$
\dot{x}(t)=\Phi x(t)+\Gamma_{1} a_{\mathrm{M}}(t)+\Gamma_{2} w(t),
$$

where

$$
\Phi=\left[\begin{array}{ccc}
0 & I_{2} & 0 \\
0 & 0 & I_{2} \\
0 & 0 & -\lambda I_{2}
\end{array}\right], \quad \Gamma_{1}=\left[\begin{array}{c}
0 \\
-I_{2} \\
0
\end{array}\right], \quad \Gamma_{2}=\left[\begin{array}{c}
0 \\
0 \\
I_{2}
\end{array}\right],
$$

and $a_{\mathrm{M}}(t)=\left[\begin{array}{ll}a_{\mathrm{M} x}(t) & a_{\mathrm{M} y}(t)\end{array}\right]^{\mathrm{T}}$ represents the missile's actual acceleration vector, $w(t)=\left[\begin{array}{ll}w_{x}(t) & w_{y}(t)\end{array}\right]^{\mathrm{T}}$ is a zeromean Gaussian white noise vector, and $\lambda$ is the reciprocal of the time constant of target acceleration. Assuming $w(t)$ remains constant in every sampling time interval, the discrete stochastic dynamic equation corresponding to Eq. (61) is given by:

$$
x_{k}=A x_{k-1}+B_{u} a_{\mathrm{M}, k-1}+B_{w} w_{k-1},
$$

where

$$
\begin{aligned}
& A=\left[\begin{array}{ccc}
I_{2} & \Delta t I_{2} & \frac{e^{-\lambda \Delta t}+\lambda \Delta t-1}{\lambda^{2}} I_{2} \\
0 & I_{2} & \frac{1-e^{-\lambda \Delta t}}{\lambda} I_{2} \\
0 & 0 & e^{-\lambda \Delta t} I_{2}
\end{array}\right] \\
& B_{u}=\left[\begin{array}{ccc}
-\frac{\Delta t^{2}}{2} I_{2} & -\Delta t I_{2} & 0
\end{array}\right]^{\mathrm{T}} \\
& B_{w}=\left[\begin{array}{c}
{\left[\left(1-e^{-\lambda \Delta t}\right) / \lambda^{3}-\Delta t / \lambda^{2}+\Delta t^{2} / 2 \lambda\right] I_{2}} \\
{\left[\left(e^{-\lambda \Delta t}-1\right) / \lambda^{2}+\Delta t / \lambda\right] I_{2}} \\
{\left[\left(1-e^{-\lambda \Delta t}\right) / \lambda\right] I_{2}}
\end{array}\right] \text {, }
\end{aligned}
$$

and $I_{2}$ is a $2 \times 2$ identity matrix, and $\Delta t$ is the time interval between measurements.

The LOS bearing measurement is:

$$
z_{\beta, k}=\beta_{k}+v_{\beta, k}=\arctan \frac{r_{x, k}}{r_{y, k}}+v_{\beta, k} .
$$

The variance of zero mean, Gaussian white measurement noise is assumed to be:

$$
R_{\beta, k}=\delta_{0}+\delta_{1} /\left(r_{x, k}^{2}+r_{y, k}^{2}\right),
$$

where $\delta_{0}$ and $\delta_{1}$ are two constants. The range measurements are given by:

$$
z_{r, k}=m_{k} r_{k}+v_{r, k},
$$

where $r_{k}=\sqrt{r_{x, k}^{2}+r_{y, k}{ }^{2}}, m_{k} \in\{0,1\}$ is the interruption sequence satisfying:

$$
\operatorname{Pr}\left[m_{k}=j \mid m_{k-1}\right]=\varepsilon_{j}, j=0,1,
$$

and $\varepsilon_{0}+\varepsilon_{1}=1$. To enhance observability in the case that range measurements are denied, a motion tracking slidingmode guidance law ${ }^{14)}$ is used to setup the trajectory in the engagement:

$$
\begin{aligned}
a_{\mathrm{M} \beta}= & (n+2)|\dot{r}|\left(\dot{\beta}-\dot{\beta}_{\mathrm{d}}\right)+2|\dot{r}| \dot{\beta}_{\mathrm{d}} \\
& +\varepsilon \operatorname{sgn}\left(\dot{\beta}-\dot{\beta}_{\mathrm{d}}\right)-r \ddot{\beta}_{\mathrm{d}},
\end{aligned}
$$

where $a_{\mathrm{M} \beta}$ represents the missile acceleration perpendicular to the LOS, $r$ and $\dot{r}$ represent the relative range and relative velocity, respectively, $\dot{\beta}_{\mathrm{d}}$ represents a given LOS angular rate of the reference signal, and $n$ and $\varepsilon$ are two constants. Generally, the reference motion is selected as $\dot{\beta}_{\mathrm{d}}=$ $\xi \sin (\omega t)$,where $\xi$ is a constant, $\omega=n_{1} \pi / t_{\mathrm{f}}, n_{1}$ is an integer, and $t_{\mathrm{f}}$ is the final time of guidance. Decomposing $a_{\mathrm{M} \beta}$ along the coordinates yields the control input of the missile:

$$
a_{\mathrm{M} \beta, k}=\left[\begin{array}{ll}
a_{\mathrm{M} \beta, k} \cos \beta_{k} & -a_{\mathrm{M} \beta, k} \sin \beta_{k}
\end{array}\right]^{\mathrm{T}} .
$$

Since universal linearization can give a more satisfactory result in angle-only tracking problems, ${ }^{11,12)}$ we utilize the universal linearization approach in the angle measurement update. Similarly, applying step-by-step Gaussian approximation, the full sequential multiple model filtering algorithm used for tracking is given in the appendix. It is, in fact, a special variant of Algorithm 1.

Consider the following initial missile-target scenario: 


$$
x_{0}=\left[\begin{array}{llllll}
3500 & 150 & -1100 & -15 & 100 & 100
\end{array}\right]^{\mathrm{T}},
$$

where the units of the relative range, relative velocity and target acceleration are supposed to be $\mathrm{m}, \mathrm{m} / \mathrm{sec}$ and $\mathrm{m} / \mathrm{sec}^{2}$, respectively. Let $\lambda=1 \mathrm{sec}^{-1}, \delta_{0}=10^{-8} \mathrm{rad}^{2} / \mathrm{sec}^{2}, \delta_{1}=$ $10^{-1} \mathrm{rad}^{2}, Q_{k}=10 \mathrm{~m} / \mathrm{sec}^{2}$ and $R_{r, k}=R_{r}=10^{2} \mathrm{~m}^{2}$. The measurement time interval is $0.02 \mathrm{sec}$. For the guidance law, we choose $\xi=0.02 \mathrm{rad} / \mathrm{sec}, t_{\mathrm{f}}=3.5 \mathrm{sec}, n_{1}=4 \mathrm{sec}$, $n=2$, and $\varepsilon=2 \mathrm{~m} / \mathrm{sec}^{2}$. For the tracking filter, the initial estimates of the mean vector and the covariance matrix of the state are taken as:

$$
\hat{x}_{0}=\left[\begin{array}{llllll}
3000 & 1200 & -950 & -100 & 0 & 0
\end{array}\right]^{\mathrm{T}}
$$

and

$$
P_{0}=\operatorname{diag}\left[\begin{array}{llllll}
10^{5} & 10^{5} & 10^{4} & 10^{4} & 10^{4} & 10^{4}
\end{array}\right],
$$

respectively. The covariance of bearing measurement noise is also estimated by the tracking filter:

$$
\hat{R}_{\beta, k}=\delta_{0}+\delta_{1} /\left(\hat{r}_{x, k}{ }^{2}+\hat{r}_{y, k}{ }^{2}\right) .
$$

The RMS relative position error estimation errors of the relative position $\tilde{r}_{k}$, relative velocity $\tilde{v}_{k}$, and target acceleration $\tilde{a}_{\mathrm{T}, k}$ are utilized to evaluate the filter's performance. These indices are computed by:

$$
\begin{aligned}
& \tilde{r}_{k}=\sqrt{\frac{\sum_{i=1}^{M}\left(r_{x, k}-\hat{r}_{x, k}^{i}\right)^{2}+\left(r_{y, k}-\hat{r}_{y, k}^{i}\right)^{2}}{M}} \\
& \tilde{v}_{k}=\sqrt{\frac{\sum_{i=1}^{M}\left(v_{x, k}-\hat{v}_{x, k}^{i}\right)^{2}+\left(v_{y, k}-\hat{v}_{y, k}^{i}\right)^{2}}{M}}
\end{aligned}
$$

and

$$
\tilde{a}_{\mathrm{T}, k}=\sqrt{\frac{\sum_{i=1}^{M}\left(a_{\mathrm{T} x, k}-\hat{a}_{\mathrm{T} x, k}^{i}\right)^{2}+\left(a_{\mathrm{T} y, k}-\hat{a}_{\mathrm{T} y, k}^{i}\right)^{2}}{M}},
$$

after $M=100$ Monte Carlo runs are carried out.

We use the Matlab software running on a personal computer (CPU: Pentium 2.4 GHz) with an MS Windows XP operation system environment to do the numerical simulation. The results indicate that the execution time in each recursion cycle is approximately $2 \mathrm{~ms}$. Two classes of cases are discussed.

Firstly, we consider deterministic interruption cases. Assume that the range measurements in the time intervals $[0.2 \mathrm{sec}, 1 \mathrm{sec}] \cup[1.5 \mathrm{sec}, 2 \mathrm{sec}] \cup[2.5 \mathrm{sec}, 3 \mathrm{sec}]$ are interrupted. In 100 Monte Carlo runs, the extended Kalman filter fails to track the target and quickly diverges. The performance of the proposed filter, however, still maintains convergent performance in the tracking scenario as it detects the interruption of the range measurements online, see Fig. 4. The reason lies in that the proposed multiple model filter has automatically abandons the interrupted measurements in the corresponding interrupted time intervals and captures
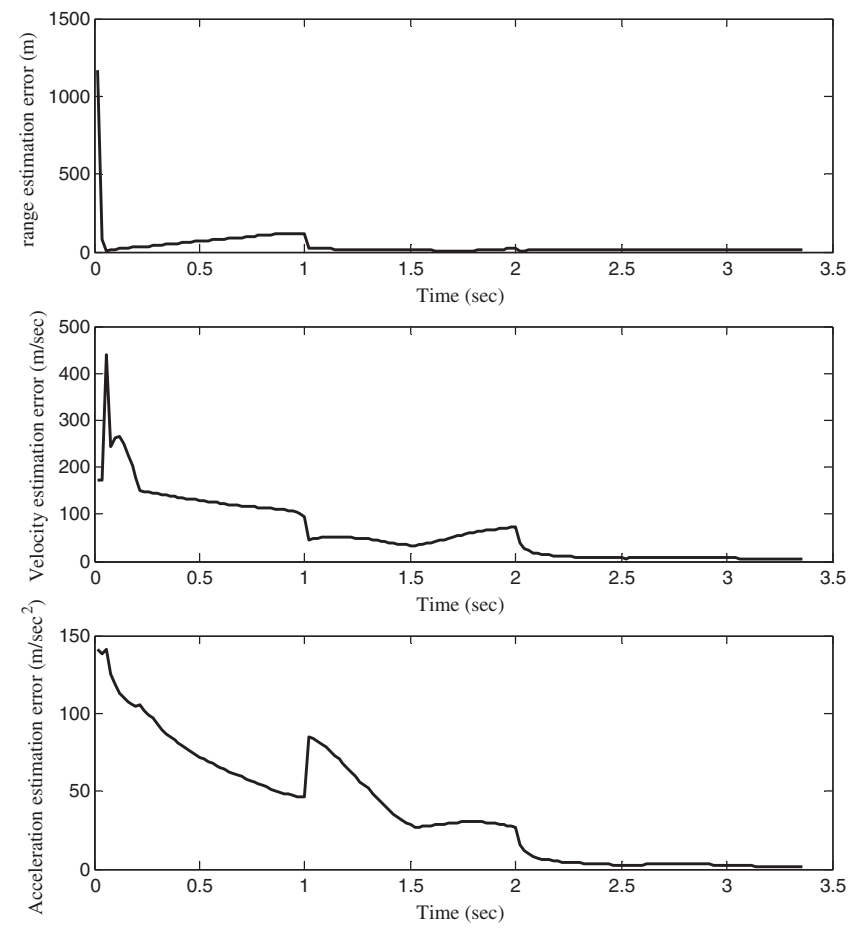

Fig. 4. RMS tracking errors in a deterministic interruption case.

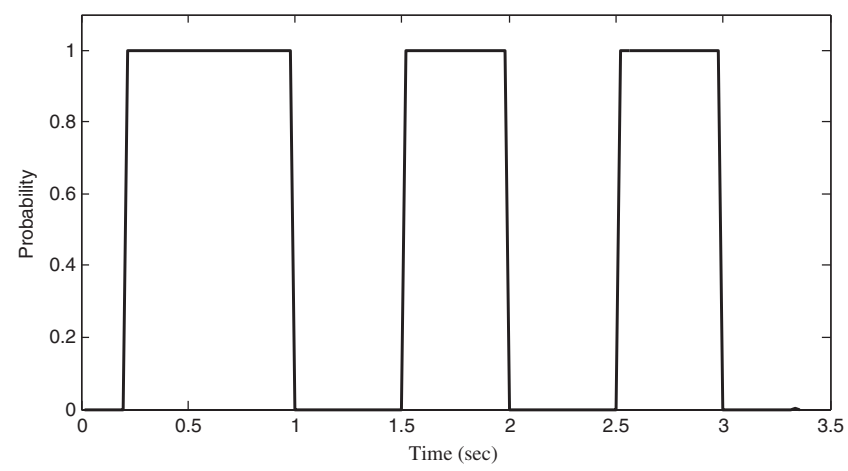

Fig. 5. Average posterior probabilities of Mode 0 in a deterministic interruption case.

the corrected range measurements in the remaining time intervals. This can be seen from the averaged posterior probability evolution curve of Mode 0 in Fig. 5. Some other deterministic cases also show that the filter has maintained effective tracking by suitable capture of the correct range measurements.

Next, we consider cases where the interruptions are generated randomly. Assume that the occurrence probability of the range measurement interruptions is $\varepsilon_{0}=0.1$ and the duration time of each interruption is $0.1 \mathrm{sec} .100$ Monte Carlo runs also show that the extended Kalman filter leads to a divergent estimation and results in a loss of tracking. In contrast, the new filter has good convergent tracking performance in random cases, see Fig. 6. The results show that the filter can work effectively under random range measurement interruptions. Note that the average posterior probabilities of range measurement interruptions have nearly the same value over the whole observation time interval due 

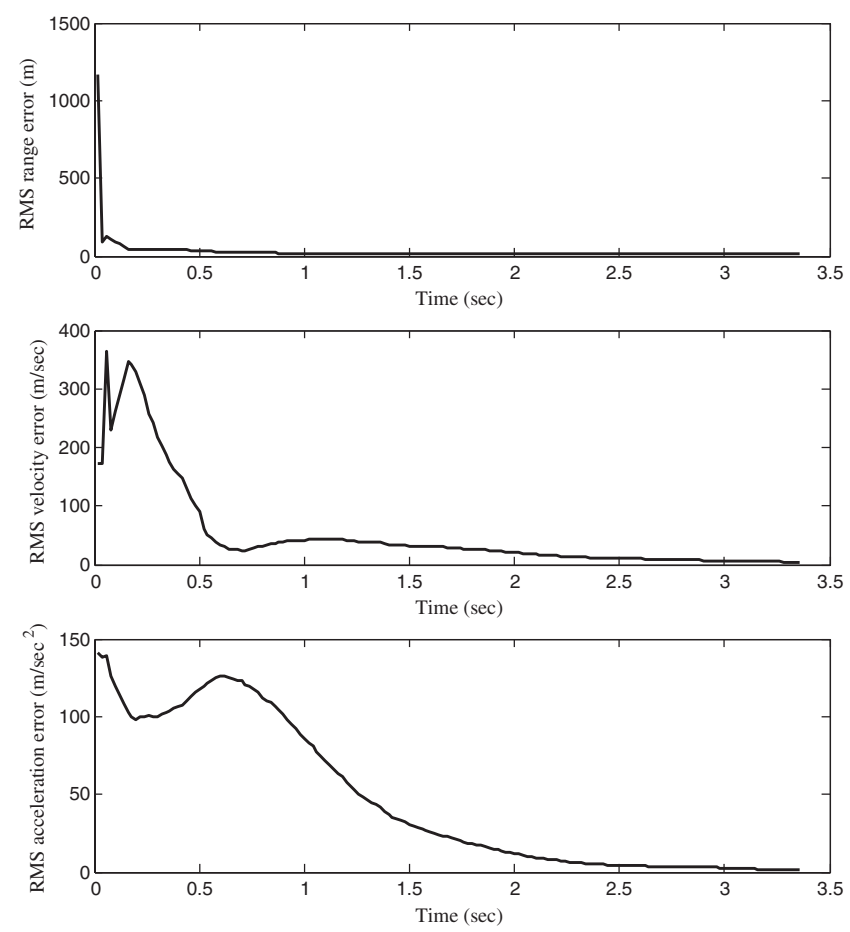

Fig. 6. RMS tracking errors in random interruption cases.

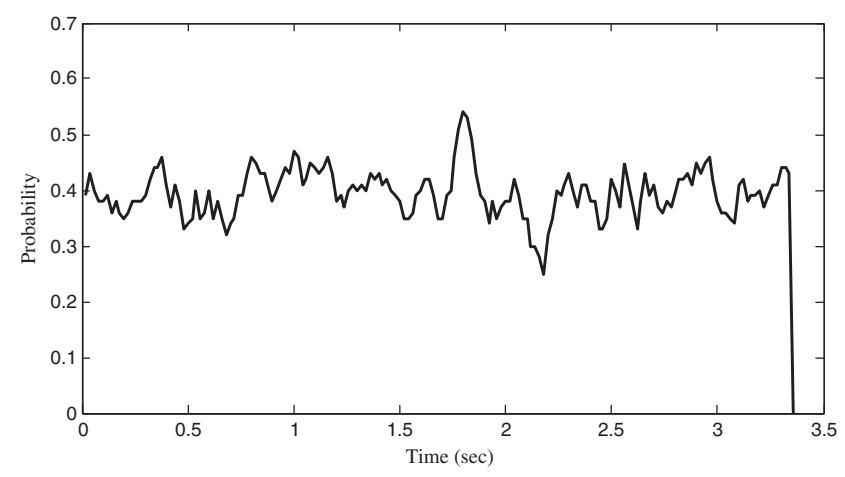

Fig. 7. Average posterior probabilities of Mode 0 in random interruption cases.

to the uniform distribution of the random interruption, as depicted in Fig. 7.

\section{Conclusions}

A new multiple model filter is proposed for discrete time stochastic systems where the measurements may possibly be inaccessible in some time intervals. Due to an inherently existing soft switching mechanism, the proposed filter can automatically abandon the denied measurements and capture the correct measurements for online state estimation. Using this mechanism, we can obtain good filtering performance. Such a method can be easily utilized in many applications, such as target tracking, failure detection and fault tolerance control, where conventional estimation methods often do not work properly. Since the multiple model filter is an approximation algorithm, there is still a possibility of divergence of the estimation. Hence, theoreti- cal analysis and experimental studies are required in the future.

\section{Acknowledgments}

This work was sponsored by the China Postdoctoral Science Foundation, and partly supported by National Natural Science Foundation of China under Grant No. 60104003 and 60374024.

\section{References}

1) Sinopoli, B., Schenato, L., Franceschetti, M., Polla, K., Jordan, M. I. and Sastry, S. S.: Kalman Filtering with Intermittent Observations, IEEE Trans. Automat. Control, 49 (2004), pp. 1453-1464.

2) Liu, X. H. and Goldsmith, A.: Kalman Filtering with Partial Observation Losses, 43rd IEEE Conference on Decision and Control, Vol. 4, 2004, pp. 4180-4186.

3) Jaffer, A. G. and Gupta, S. C.: Optimal Sequential Estimation of Discrete Time Processes with Markov Interrupted Observations, IEEE Trans. Automat. Control, 16 (1971), pp. 471-475.

4) Sawaragi, Y., Katayama, T. and Fujishige, S.: Adaptive Estimation for a Linear System with Interrupted Observation, IEEE Trans. Automat. Control, 16 (1973), pp. 152-154.

5) Hadidi, M. T. and Schwartz, S. C.: Linear Recursive State Estimators under Uncertain Observations, IEEE Trans. Automat. Control, 24 (1979), pp. 944-948.

6) Blackman, S. S. and Popoli, R.: Design and Analysis of Modern Tracking Systems, Artech House, Norwood, MA, 1999, pp. 259-319.

7) Frina, A.: Target Tracking with Bearings-Only Measurements, Signal Process., 78 (1999), pp. 61-78.

8) Zhang, Y.-A., Zhou, D. and Duan, G.-R.: Simplified Multiple Model Filtering of Target Glint, Proceedings of the 5th World Congress on Intelligent Control and Automation, Vol. 2, 2004, pp. 1606-1609.

9) Zhang, Y.-A., Zhou, D. and Duan, G.-R.: Multiple Model Filtering in the Presence of Gaussian Mixture Measurement Noises, Chinese J. Aeronaut., 17 (2004), pp. 229-234.

10) Bar-Shalom, Y., Li, X.-R. and Kirubarajan, T.: Estimation with Applications to Tracking and Navigation: Theory, Algorithms and Software, John Wiley \& Son, Inc., New York, 2001, pp. 421-488.

11) Pachter, M. and Chandlser, P. R.: Universal Linearization Concept for Extended Kalman Filtering, IEEE Trans. Aero. Elec. Sys., 29 (1993), pp. 946-962.

12) Song, T. L. and Speyer, J. L.: A Stochastic Analysis of a Modified Gain Extended Kalman Filter with Applications to Estimation with Bearings Only Measurements, IEEE Trans. Automat. Control, 30 (1985), pp. 490-949.

13) Li, X.-R. and Zhang, Y.-M.: Numerically Robust Implementation of Multiple-Model Algorithms, IEEE Trans. Aero. Elec. Sys., 36 (2000), pp. 266-278.

14) Zhou, D. and Mu, C.-D.: Motion Tracking Sliding-Mode Guidance of a Homing Missile with Bearings Only Measurements, Trans. Jpn. Soc. Aeronaut. Space Sci., 43 (2000), pp. 130-136.

\section{Appendix}

This appendix gives the full algorithm of the sequential multiple model filter for target tracking with intermittent range measurements. Notice that the universal linearization approach $^{11,12)}$ is used for the angle measurement update. Since the occurrence of the range measurement interruption has a stationary distribution with respect to time, we need not calculate the predicted probability of the modal state. ${ }^{8)}$

\section{Algorithm 2.}

1) Initialization. Pre-specify the original a priori estimates of the mean and covariance matrix of the state: 
$\left\{\hat{x}_{0 \mid 0}, P_{0 \mid 0}\right\}$.

2) Recursive estimation. $k=1,2, \cdots$

a) Time update:

$$
\begin{gathered}
\hat{x}_{k \mid k-1}=A_{k} \hat{x}_{k-1 \mid k-1}+B_{u} a_{\mathrm{M}, k-1} \\
P_{k \mid k-1}=A_{k} P_{k-1 \mid k-1} A_{k}^{\mathrm{T}}+B_{k} Q_{k} B_{k}^{\mathrm{T}} \\
\hat{z}_{r, k \mid k-1}=\sqrt{\hat{r}_{x, k \mid k-1}{ }^{2}+\hat{r}_{y, k \mid k-1}{ }^{2}} \\
\hat{z}_{\beta, k \mid k-1}=\arctan \frac{\hat{r}_{x, k \mid k-1}}{\hat{r}_{y, k \mid k-1}} .
\end{gathered}
$$

b) Angle measurement update:

$$
\begin{aligned}
& H_{\beta, k}=\left[\begin{array}{llllll}
\frac{\cos \hat{z}_{\beta, k \mid k-1}}{\hat{r}_{k \mid k-1}} & -\frac{\sin \hat{z}_{\beta, k \mid k-1}}{\hat{r}_{k \mid k-1}} & 0 & 0 & 0 & 0
\end{array}\right] \\
& v_{\beta, k}=z_{\beta, k}-\hat{z}_{\beta, k \mid k-1} \\
& S_{\beta, k}=H_{\beta, k} P_{k \mid k-1} H_{\beta, k}^{\mathrm{T}}+R_{\beta, k} \\
& K_{\beta, k}=P_{k \mid k-1} H_{\beta, k}^{\mathrm{T}} S_{\beta, k}^{-1} \\
& \bar{x}_{k \mid k}=\hat{x}_{k \mid k-1}+K_{\beta, k} v_{\beta, k}
\end{aligned}
$$

$$
\begin{gathered}
g\left(z_{\beta, k}\right)=\frac{v_{\beta, k}}{\hat{r}_{k \mid k-1} \sin v_{\beta, k}} H_{\beta, k} \\
\bar{P}_{k \mid k}=\left[I-K_{\beta, k} g\left(z_{\beta, k}\right)\right] P_{k \mid k-1}\left[I-K_{\beta, k} g\left(z_{\beta, k}\right)\right]^{\mathrm{T}} \\
+K_{\beta, k} R_{\beta, k} K_{\beta, k}{ }^{\mathrm{T}} .
\end{gathered}
$$

c) Range measurement update: When $m_{k}=0$, we have

$$
H_{r, k}^{0}=0, v_{r, k}^{0}=z_{r, k}-0=z_{r, k}, S_{r, k}^{0}=R_{r, k}, K_{r, k}^{0}=0
$$

$$
\begin{gathered}
\hat{x}_{k \mid k}^{0}=\bar{x}_{k \mid k}+K_{r, k}^{1} v_{r, k}^{0}=\bar{x}_{k \mid k} \\
P_{k \mid k}^{0}=\bar{P}_{k \mid k} \\
L_{r, k}^{0}=\frac{1}{\sqrt{\operatorname{det}\left(2 \pi S_{r, k}^{0}\right)}} \exp \left[-\frac{1}{2} v_{r, k}^{0 \mathrm{~T}} S_{r, k}^{0}{ }^{-1} v_{r, k}^{0}\right] \\
=\frac{1}{\sqrt{\operatorname{det}\left(2 \pi R_{r, k}\right)}} \exp \left[-\frac{1}{2} z_{r, k}^{\mathrm{T}} R_{r, k}^{-1} z_{r, k}\right] .
\end{gathered}
$$

When $m_{k}=1$, we have

$$
\begin{aligned}
& H_{r, k}^{1}=\left[\begin{array}{llllll}
\bar{r}_{x, k} / \sqrt{\bar{r}_{x, k}{ }^{2}+\bar{r}_{y, k}{ }^{2}} & \bar{r}_{y, k} / \sqrt{\bar{r}_{x, k}^{2}+\bar{r}_{y, k}{ }^{2}} & 0 & 0 & 0 & 0
\end{array}\right] \\
& v_{r, k}^{1}=z_{r, k}-\sqrt{\bar{r}_{x, k}^{2}+\bar{r}_{y, k}^{2}} \\
& S_{r, k}^{1}=H_{r, k}^{1} \bar{P}_{k \mid k} H_{r, k}^{1 \mathrm{~T}}+R_{r, k} \\
& K_{r, k}^{1}=\bar{P}_{k \mid k} H_{r, k}^{1}{ }^{\mathrm{T}} S_{r, k}^{1}{ }^{-1} \\
& \hat{x}_{k \mid k}^{1}=\bar{x}_{k \mid k}+K_{r, k}^{1} v_{r, k}^{1} \\
& P_{k \mid k}^{1}=\left(I-K_{r, k}^{1} H_{r, k}^{1}\right) \bar{P}_{k \mid k}\left(I-K_{r, k}^{1} H_{r, k}^{1}\right)^{\mathrm{T}} \\
& +K_{r, k}^{1} R_{r, k} K_{r, k}^{1 \mathrm{~T}} \\
& L_{r, k}^{1}=\frac{1}{\sqrt{\operatorname{det}\left(2 \pi S_{r, k}^{1}\right)}} \exp \left[-\frac{1}{2} v_{r, k}^{1} S_{r, k}^{1}{ }^{-1} v_{r, k}^{1}\right] . \\
& \mu_{k}^{j}=\operatorname{Pr}\left(m_{k} \mid Z^{k}\right)=\frac{L_{r, k}^{j} \varepsilon_{j}}{c_{k}}, \\
& c_{k}=\sum_{j=0}^{1} L_{r, k}^{j} \varepsilon_{j} . \\
& \hat{x}_{k \mid k}=\sum_{j=0}^{1} \mu_{k}^{j} \hat{x}_{k \mid k}^{j} \\
& P_{k \mid k}=\sum_{j=0}^{1} \mu_{k}^{j}\left[P_{k \mid k}^{j}+\left(\hat{x}_{k \mid k}^{j}-\hat{x}_{k \mid k}\right)\left(\hat{x}_{k \mid k}^{j}-\hat{x}_{k \mid k}\right)^{\mathrm{T}}\right] .
\end{aligned}
$$

Modal state posterior probability computation: 